\title{
Coordination Cages as Permanently Porous Ionic Liquids
}

Lillian Ma ${ }^{1}$, Cally J. E. Haynes ${ }^{1,2}$, Angela B. Grommet ${ }^{1}$, Anna Walczak ${ }^{3,4}$, Christopher C. Parkins ${ }^{1}$, Cara M. Doherty $^{5}$, Louis Longley ${ }^{6}$, Arnaud Tron ${ }^{1}$, Artur R. Stefankiewicz ${ }^{3,4}$, Thomas D. Bennett ${ }^{6 *}$, and Jonathan R. Nitschke ${ }^{1 *}$

${ }^{1}$ University of Cambridge, Department of Chemistry, Lensfield Road, Cambridge, UK, CB2 1EW. E-mail: jrn34@cam.ac.uk

${ }^{2}$ Current address: University College London, Department of Chemistry, 20 Gordon Street, London, UK, WC1H 0AJ.

${ }^{3}$ Center for Advanced Technologies, Adam Mickiewicz University, ul. Uniwersytetu Poznańskiego 10, 61614 Poznań, PL

${ }^{4}$ Faculty of Chemistry, Adam Mickiewicz University, ul. Uniwersytetu Poznańskiego 8, 61-614 Poznań, PL

${ }^{5}$ Manufacturing, Commonwealth Scientific and Industrial Research Organisation, Clayton South, VIC 3169, Australia

${ }^{6}$ University of Cambridge, Department of Materials Science and Metallurgy, Charles Babbage Road, Cambridge, UK, CB3 OFS

*To whom correspondence should be addressed: tdb35@cam.ac.uk, jrn34@cam.ac.uk

\begin{abstract}
Porous materials are widely used in industry for applications that include chemical separations and gas scrubbing. These materials are typically porous solids, though the liquid state can be easier to manipulate in industrial settings. The idea of combining the size- and shape-selectivity of porous domains with the fluidity of liquids is a promising one and porous liquids composed of functionalized organic cages have recently attracted attention. Here, we describe an ionic-liquid, porous, tetrahedral coordination cage. Complementing the gas-binding observed in other porous liquids, this material also encapsulates non-gaseous guests - shape- and size-selectivity was observed for a series of alcohol isomers. Three gaseous guests, chlorofluorocarbons CFC-11, CFC-12, and CFC-13, were also shown to be taken up by the liquid coordination cage with an affinity increasing with their size. We hope that these findings will lead to the synthesis of other porous liquids whose guest-uptake properties may be tailored to fulfil specific functions.
\end{abstract}

Recent work has shown that persistent cavities can be engineered into liquids, lending them permanent porosity. These new materials were initially proposed by James in $2007^{1}$, who recognised three distinct types of them. The simplest of these, Type I permanently porous liquids, consist of rigid hosts with empty cavities that are liquid in their neat state $^{2,3}$, without requiring an additional solvent for fluidity ${ }^{4-7}$. Metalorganic frameworks (MOFs) have also been observed to form liquid phases that are inferred to be porous ${ }^{8,9}$, although the high temperatures required preclude guest binding.

Previously reported examples of porous liquids have included surface-modified hollow silica spheres ${ }^{2}$ and hollow carbon spheres ${ }^{3}$, crown ether-functionalised organic cages ${ }^{5}$, and dispersions ${ }^{4,6}$ or slurries ${ }^{7}$ of porous framework materials in ionic liquids. To date, applications of these materials have focussed on gas storage and separation ${ }^{2,10,11}$. However, we are not aware of the binding of guest molecules larger than carbon dioxide or methane inside the cavities of porous liquids, restricting the potential application of these 
materials. The development of a porous liquid with cavities that host more diverse guest molecules is thus an attractive goal.

Here we report a liquid coordination cage, obtained by incorporating PEG-imidazolium chains into the periphery of parent $\mathrm{Zn}_{4} \mathrm{~L}_{4}$ tetrahedron 1 (Fig. 1a) to yield cage 2 which is a liquid in the neat state. This porous liquid sequesters trichlorofluoromethane $\left(\mathrm{CFCl}_{3}, \mathrm{CFC}-11\right)$, dichlorodifluoromethane $\left(\mathrm{CF}_{2} \mathrm{Cl}_{2}, \mathrm{CFC}\right.$ $12)$, and chlorotrifluoromethane $\left(\mathrm{CF}_{3} \mathrm{Cl}, \mathrm{CFC}-13\right)$, which are ozone-depleting and climate-warming chlorofluorocarbons amongst the most abundant and long-lived in the atmosphere ${ }^{12}$. Furthermore, cage 2 represents a porous liquid capable of binding non-gaseous molecules: larger guests, such as butanol and propanol isomers, were encapsulated within $\mathbf{2}$, with binding selectively driven by size and shape fit. Neat liquid cage $\mathbf{2}$ has negligible vapour pressure, which means that it may be recycled by removing encapsulated guests under vacuum.

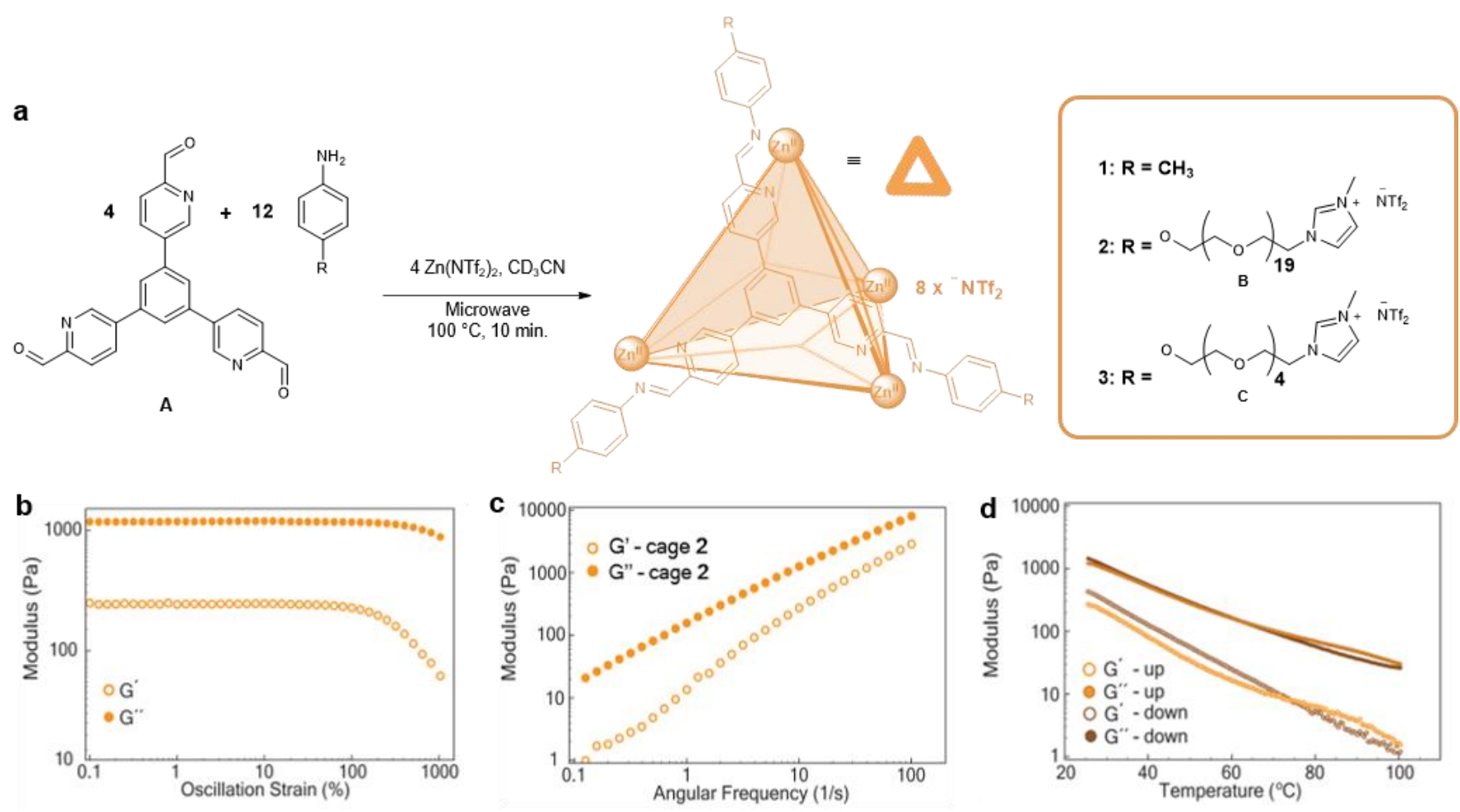

Figure 1 | Preparation and rheology of cages 1-3. a, Previously published tetrahedral cage 1 can be self-assembled when trialdehyde $\mathbf{A}$ and aniline subcomponents were combined with $\mathrm{Zn}\left(\mathrm{NTf}_{2}\right)_{2}$ in $\mathrm{CH}_{3} \mathrm{CN}$. Cage 2 formed when a PEG-imidazolium functionalised aniline $\mathbf{B}$ was employed in place of $\mathbf{A}$; as cage $\mathbf{3}$ formed from pentaethylene-glycol imidazolium aniline $\mathbf{C}$. Characterisation of cages 1-3 is presented in SI Section 1.4. b, Dynamic rheological analysis of neat liquid cage 2. Oscillatorydependent rheology of neat liquid cage 2 at $25^{\circ} \mathrm{C}$ showed liquid behaviour with $\mathrm{G}^{\prime \prime}$ (loss modulus) greater than $\mathrm{G}^{\prime}$ (storage modulus) throughout the measurement. c, Frequency dependent oscillatory rheology measurement indicating that neat liquid cage 2 remained fluid under all measured frequency regimes $\left(\mathrm{G}^{\prime \prime}>\mathrm{G}^{\prime}\right)$. d, Oscillatory temperature ramps at $5{ }^{\circ} \mathrm{C}$ min ${ }^{-1}$ measured at 10 $\operatorname{rad~s}^{-1}$ and $1 \%$ strain between $25-100{ }^{\circ} \mathrm{C}$, showing that the viscosity of neat liquid cage 2 decreased as temperature increased. Additional rheological experiments can be found in SI Section 6.

\section{Results and discussion}

Cage 2 was designed with two goals in mind: firstly, to synthesise a metal-organic capsule that is liquid at room temperature in the neat state, and secondly, to ensure that the host cavities remained empty and accessible. The robust framework of $\mathbf{1}^{13}$ was thus functionalised with 12 poly(ethylene glycol) chains to render the cage a liquid at room temperature after removing the solvent. Positive imidazolium moieties were 
chosen to terminate the PEG chains, so that coulombic repulsion may keep the chains from entering the positively charged cage cavity. Bis(trifluoromethane)sulfonamide (triflimide, $\mathrm{NTf}_{2}{ }^{-}$) was chosen as the counterion as previous studies ${ }^{13}$ have shown that $\mathrm{NTf}_{2}{ }^{-}$is too large to bind inside ${ }^{13}$ the isostructural cavity of cage 1.

\section{Synthesis and characterisation of permanently porous liquid cage 2.}

Cage 2 was prepared via the reaction of trialdehyde A (4 equiv.) and PEG-imidazolium aniline B (12 equiv.), with zinc(II) triflimide (4 equiv.) in $\mathrm{CH}_{3} \mathrm{CN}$ (Fig. 1a). Its formation was confirmed by ${ }^{1} \mathrm{H}$ and ${ }^{19} \mathrm{~F}$ NMR in $\mathrm{CD}_{3} \mathrm{CN}$ (SI Section 1.4). The close match between the solution-phase ${ }^{1} \mathrm{H}$ NMR spectra of cages 1 and 2 (Supplementary Figs. 65-66) confirm that the PEG chains functionalising cage 2 did not occupy the pores of the capsule. We were unable to characterise cage $\mathbf{2}$ using electrospray ionization mass spectrometry (ESI-MS) because the cage was unstable under the required ionization conditions.

Isostructural cage $\mathbf{3}$ was therefore prepared from $\mathbf{C}$, an analogue of $\mathbf{B}$ with a pentaethylene glycol chain. Unlike 2, cage $\mathbf{3}$ was a solid at room temperature after the solvent was removed, demonstrating that longer PEG chains were essential to lower the melting point of the neat cage below room temperature. Because the aromatic ${ }^{1} \mathrm{H}$ NMR signals from the core of cage 3 in $\mathrm{CD}_{3} \mathrm{CN}$ solution appeared at the same chemical shift values as the signals from cage $\mathbf{2}$ (Supplementary Fig. 74), we inferred that cages $\mathbf{2}$ and $\mathbf{3}$ contain the same tetrahedral framework. Unlike 2, however, 3 was observable by ESI-MS (Supplementary Fig. 1), confirming its $\mathrm{Zn}_{4} \mathrm{~L}_{4}$ composition, as well as reinforcing our assignment of the structure of 2 .

After cage 2 was synthesised and characterised in acetonitrile solution, the solvent was removed under dynamic vacuum. This neat ionic liquid cage 2 was characterised by variable temperature ${ }^{1} \mathrm{H}$ NMR (VTNMR) at $70{ }^{\circ} \mathrm{C}$; because the neat liquid cage is viscous, analysis at higher temperature was found to improve cage tumbling and thus signal resolution within the NMR sample. The ${ }^{1} \mathrm{H}$ and ${ }^{19} \mathrm{~F}$ NMR signals for neat liquid cage 2 were thus broadened, but matched the values observed in $\mathrm{CD}_{3} \mathrm{CN}$ solution (Supplementary Figs. 63, 68).

Positron annihilation lifetime spectroscopy (PALS) was used to probe the porosity of neat liquid cage 2 (SI Section 4). This technique detects voids within materials by probing the free volume with a positron source, such as ${ }^{22} \mathrm{Na}$. This process results in the formation ortho-positronium (o-Ps), a parallel spin complex between $e^{+}$and $e^{-}$. The average pore diameter of the sample is correlated to the lifetime of the $o$-Ps because larger pores correlate with slower decay rates ${ }^{14}$. The $o$-Ps lifetime for neat liquid cage 2 was measured to be $2.34 \pm 0.05 \mathrm{~ns}$, which corresponds to an average void diameter of $6.29 \pm 0.08 \AA$ (Supplementary Table 1). VOIDOO calculations ${ }^{15}$ performed on the crystal structure of analogous cage 1 yielded a volume of $130 \AA^{3}$, which corresponds to a spherical diameter of $6.28 \AA$ (SI Section 4$)^{13}$. The close agreement between the expected pore diameter of the cage and the experimental value derived from PALS supports the hypothesis that the hollow cage scaffold of cage $\mathbf{2}$ is intact and empty in the neat state.

To probe its stability and phase transition behaviour, neat liquid cage $\mathbf{2}$ was characterised by differential scanning calorimetry (DSC) and thermogravimetric analysis (TGA) (Supplementary Figs. 11, 12). DSC 
data indicated that the cage underwent a reversible phase change with an onset temperature of $-44{ }^{\circ} \mathrm{C}$, implying solidification below this temperature. The TGA trace of neat liquid cage $\mathbf{2}$ showed no significant loss of mass between room temperature and $300{ }^{\circ} \mathrm{C}$, above which decomposition was observed. The DSC scan of solid cage 3 showed no evidence of phase transitions below decomposition at $340{ }^{\circ} \mathrm{C}$ (Supplementary Fig. 13).

In addition to DSC/TGA, the rheological properties of neat liquid cage 2 were studied through oscillatory strain measurements over the range of $0.1-1000 \%$ at a fixed angular frequency of $10 \mathrm{rad} \mathrm{s}^{-1}$ (Fig. 1b). Above $100 \%$ strain, the $\mathrm{G}^{\prime}$ value (loss modulus) dramatically decreased but the material continued to exhibit fluid-like behaviour. Moreover, a frequency sweep at $1 \%$ strain (Fig. 1c) indicated a $\mathrm{G}^{\prime}$ consistent with fluid-like behaviour that depended strongly upon frequency, with more than 3 orders of magnitude change in $\mathrm{G}^{\prime}$ over the probed frequency range. To further investigate the temperature-dependent behaviour, a temperature sweep was performed at $1 \%$ strain and $10 \mathrm{rad} \mathrm{s}^{-1}$, highlighting the fluidity of 2 at temperatures ranging from $20-100{ }^{\circ} \mathrm{C}$, and showing that the viscosity of neat liquid cage $\mathbf{2}$ decreased as temperature increased (Fig. 1d). The thixotropic flow ramp cycle indicated deviation between the up- and down-shearing cycles, signifying a time-dependent restructuring of this viscous fluid (Supplementary Figs. 15-16).

\section{Guest selectivity of cage 2 .}

Cage 2 was combined with different propanol and butanol isomers to investigate the selectivity of guest binding as a function of size and branching patterns. Screening for guest binding was initially performed in $\mathrm{CD}_{3} \mathrm{CN}$ solution to probe the size and shape selectivity of cage 2 (Fig. 2a, Figs. 79-84), by adding $10 \mu \mathrm{L}$ of each guest to $0.5 \mathrm{~mL}$ of a $2 \mathrm{mM}$ solution of cage 2 (100-fold excess). The results indicated the encapsulation of isomers of propanol and butanol within cage 2, with affinities that depend upon the structure of the guest.. Guest binding for cage 2 in the neat liquid state was then monitored using ${ }^{1} \mathrm{H}$ NMR at $70{ }^{\circ} \mathrm{C}$ (Fig. 2b, Supplementary Figs. 1920). The set up used for this experiment is shown in Fig. 2c and described in detail in the SI section 7.4. Under solvent-free conditions, neat liquid cage $\mathbf{2}$ showed similar guest-binding preferences to cage 2 dissolved in $\mathrm{CD}_{3} \mathrm{CN}$. This trend is summarised in Fig. 2 d. Upon the addition of $t$-butanol, $i$-butanol, or $i$ propanol, the ${ }^{1} \mathrm{H}$ NMR spectra of neat liquid cage 2 showed peaks attributed to the encapsulated guest molecules that were shifted upfield compared to the free guests, with chemical shifts below 0 ppm (Fig. 2b, Supplementary Figs. 19-20), which confirmed that these guests could be encapsulated. In contrast, when $n$ propanol or $s$-butanol were combined with the neat liquid cage 2 , the ${ }^{1} \mathrm{H}$ NMR signals corresponding to encapsulated alcohols were relatively smaller (Fig. 2b, Supplementary Figs. 19-20), indicating a lesser degree of encapsulation and hence weaker binding. Comparison of the integrated intensities of bound guest signals to cage framework peaks thus allowed us to gauge that $n$-propanol and $s$-butanol were bound more weakly than $t$-butanol, $i$-butanol and $i$-propanol. The NMR results also indicated that $n$-butanol did not interact significantly with neat liquid cage 2 (Fig. 2b, Supplementary Figs. 19-20). This trend in solvent-free binding was consistent with the behaviour of cage 2 in $\mathrm{CD}_{3} \mathrm{CN}$ solution (Fig. 2a, SI Section 7.2). 


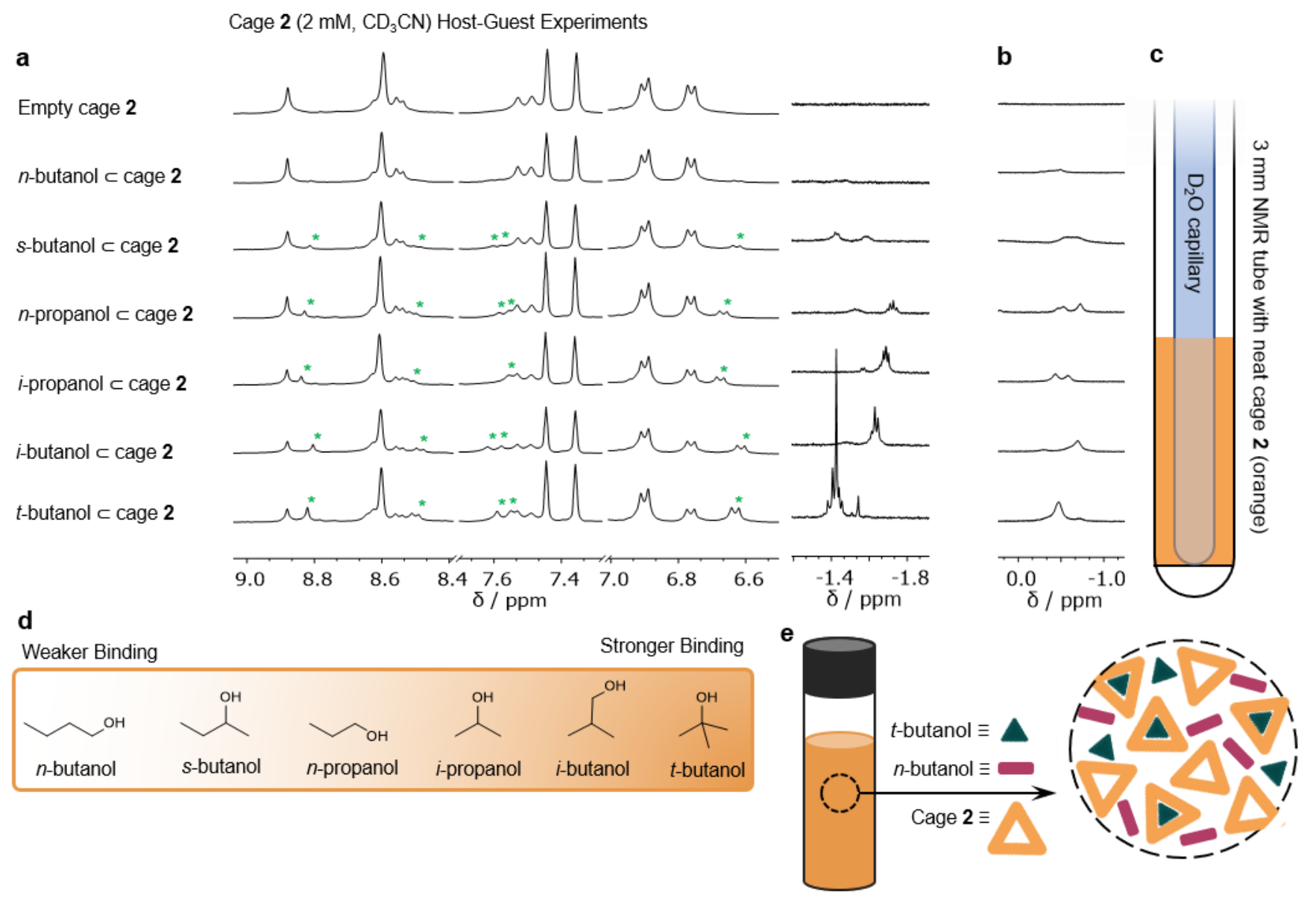

Figure 2 | Shape selectivity in the encapsulation of isomers of propanol and butanol by cage 2. a, Partial solution-phase ${ }^{1} \mathrm{H}$ NMR spectra $\left(298 \mathrm{~K}, 400 \mathrm{MHz}, \mathrm{CD}_{3} \mathrm{CN}\right)$ of the complexes of cage 2 incorporating the alcohols shown. Host-guest peaks in the aromatic region are labelled with an asterisk (*) and all peaks below 0 ppm are assigned to the internally-bound guest. $\mathbf{b}$, Partial solvent-free variable temperature ${ }^{1} \mathrm{H}$ NMR spectra $(343 \mathrm{~K}, 400 \mathrm{MHz})$ of complexes of neat liquid cage 2 with 30 wt\% of the alcohols shown in d, taken with the tube assembly depicted in c (30 wt\% guest). c, In order to record NMR spectra under solventfree conditions, neat liquid cage 2 was loaded into a $3 \mathrm{~mm}$ NMR tube containing a capillary filled with $\mathrm{D}_{2} \mathrm{O}$ to provide an NMR lock signal. d, Cage $\mathbf{2}$ bound a range of different propanol and butanol isomers; a better shape match between guest and host cavity led to stronger binding. e, Neat liquid cage $\mathbf{2}$ selectively encapsulated $t$-butanol over $n$-butanol.

Based on these guest binding preferences, we hypothesised that branched alcohols might present a better shape match for the cavity of cage 2 than their linear isomers, leading to stronger binding (Fig. 2d). In experiments where equivalent amounts of $n$-butanol and $t$-butanol had both been added to cage 2 , after equilibration at $298 \mathrm{~K}$ for $18 \mathrm{~h}$, only encapsulated $t$-butanol could be observed by ${ }^{1} \mathrm{H}$ NMR in both $\mathrm{CD}_{3} \mathrm{CN}$ solution and in the neat liquid state (Fig. 2e, Supplementary Figs. 16-17, 87). We also discovered that encapsulated guests were removed from cage 2 under vacuum (1.35 mbar), thus enabling neat liquid cage 2 to be recovered and recycled without further purification. To investigate the recyclability of $\mathbf{2}$, a sample of $t$ butanol $\subset \mathbf{2}$ was exposed to vacuum for $1 \mathrm{~h}$. The recovered material was then analysed by solution-phase ${ }^{1} \mathrm{H}$ NMR, which indicated that $t$-butanol had been removed, and that cage 2 remained intact. The host-complex was then regenerated via the addition of $t$-butanol, and this cycle was repeated five times, with no cage decomposition (Supplementary Figs. 25, 106).

\section{Encapsulation of CFCs in neat liquid cage 2.}

Trichlorofluoromethane $\left(\mathrm{CFCl}_{3}, \mathrm{CFC}-11\right)$, dichlorodifluoromethane $\left(\mathrm{CF}_{2} \mathrm{Cl}_{2}, \mathrm{CFC}-12\right)$, and chlorotrifluoromethane $\left(\mathrm{CF}_{3} \mathrm{Cl}, \mathrm{CFC}-13\right)$ are three of the most abundant and long-lived ozone depleting 
agents, with one gram of each in the atmosphere being equivalent to 4750, 10900, and 14400 grams of $\mathrm{CO}_{2}$, respectively, in global warming effects ${ }^{12} . \mathrm{CFCl}_{3}$ alone contributes $25 \%$ of all chlorine reaching the stratosphere. Although $\mathrm{CFCl}_{3}$ emissions had been declining consistently since the late 1980s, an unexpected but steady increase of this environmental contaminant began in 2012 and continues today ${ }^{16,17}$. Recovery of the ozone layer is dependent on a sustained reduction in the emissions of these three CFCs. MOFs ${ }^{18}$, ionic liquids $^{19}$, and anion cages ${ }^{20}$ have all been shown to adsorb, dissolve or encapsulate CFCs (see SI Section 12). However, novel means of selectively and reversibly binding these CFCs could lead to new processes for their remediation.

We thus investigated the uptake and binding of these three CFCs within cage 2 by ${ }^{1} \mathrm{H}$ and ${ }^{19} \mathrm{~F} \mathrm{NMR}$ using neat liquid cage $\mathbf{2}$ and in $\mathrm{CD}_{3} \mathrm{CN}$ solution. We were unable to analyse the neat liquid $\mathrm{CFC} \subset \mathbf{2}$ samples using the NMR protocol developed for the alcohol guests (Fig. 2c) due to the volatility of the CFCs, which resulted in $\mathrm{CFC}$ evaporation upon heating the samples to $70^{\circ} \mathrm{C}$. Therefore, samples of neat liquid cage 2 were exposed to gaseous CFCs as shown in Fig. 3a and dissolved in $\mathrm{CD}_{3} \mathrm{CN}$ immediately prior to analysis using standard solution-phase NMR protocols. The resulting spectra were consistent with the results obtained after the addition of the CFCs to cage 2 in $\mathrm{CD}_{3} \mathrm{CN}$ solution after equilibration for $18 \mathrm{~h}$ (Fig. 3b-c, Supplementary Figs. 92-103). Signals corresponding to encapsulated CFCs were observed in all cases in the ${ }^{19} \mathrm{~F}$ NMR spectra of the CFC host-guest experiments, confirming that porous liquid cage $\mathbf{2}$ solubilised and encapsulated all three CFCs. A kinetic study of CFC uptake by cage $\mathbf{2}$ in $\mathrm{CD}_{3} \mathrm{CN}$ solution indicated that the degree of encapsulation observed in the neat liquid cage experiments could not have been achieved in the short time between dissolving the cage and the NMR analysis (see SI Section 8.5), hence we infer that cage 2 had encapsulated the CFCs in the neat liquid state prior to dissolution, and with an order of affinity $\mathrm{CFCl}_{3}>\mathrm{CF}_{2} \mathrm{Cl}_{2}>\mathrm{CF}_{3} \mathrm{Cl}$, as in $\mathrm{CD}_{3} \mathrm{CN}$ solution.

Cage 2 displayed differing binding affinities for the three CFCs investigated. Based on the integration of the free and encapsulated guest peaks, we estimated that $\mathrm{CFCl}_{3}$ was bound the most strongly, followed by $\mathrm{CF}_{2} \mathrm{Cl}_{2}$ and $\mathrm{CFCl}_{3}$ (Fig. 3b, 3c, SI Section 8.4). This ordering tracks the sizes of the CFCs; we infer that the largest, $\mathrm{CFCl}_{3}$, presents the best size and shape match for the cavity of cage $\mathbf{2}$. In contrast with previously reported host-guest experiments conducted with anionic cages in acetonitrile solution ${ }^{20}$, our material functions as its own solvent, enabling solvent-free conditions. Hence, cage $\mathbf{2}$ combines the benefits of ionic liquids and cages by mediating the dissolution and encapsulation of CFCs within a singlecomponent fluid phase, thus providing a new dimension of selectivity

As with the alcohols discussed above, all three CFCs could also be removed from cage 2 under reduced pressure, and the cage could be recycled five times without evidence of cage decomposition (Fig. 3d, SI Section 8.6, Supplementary Figs. 104-105). This cyclic catch-and-release binding may thus serve as the basis for selectively trapping CFCs in the context of a recycling or remediation process. 

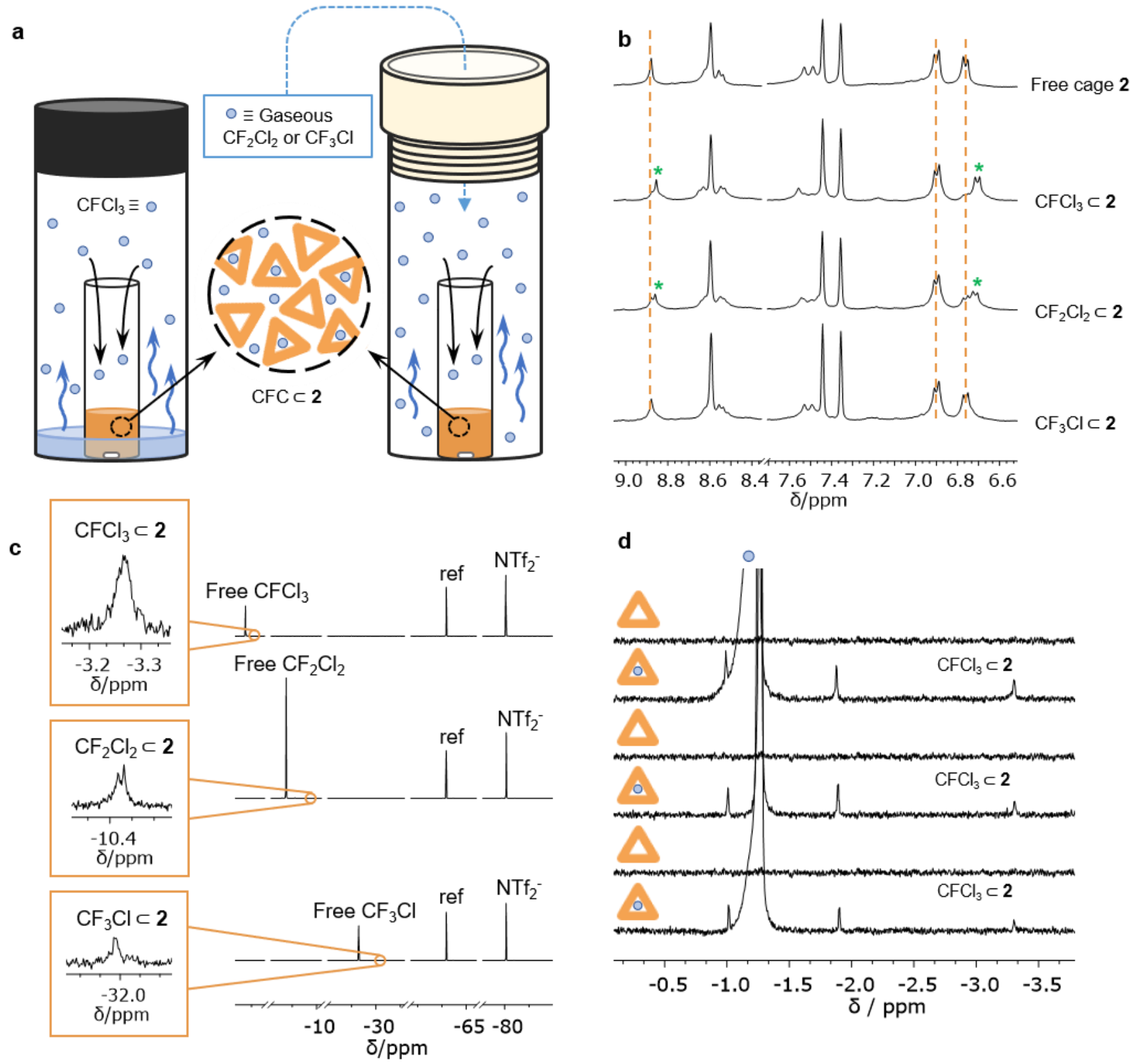

d

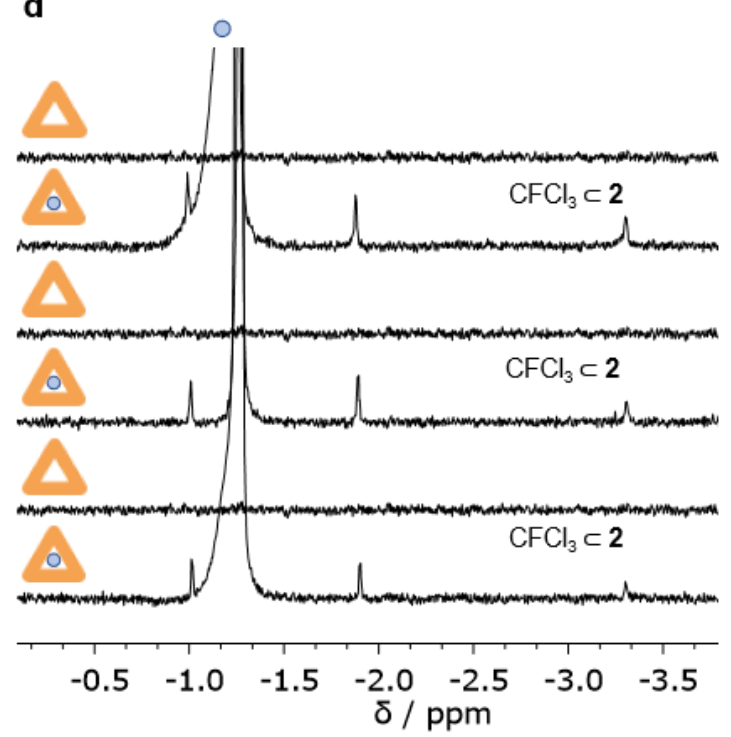

Figure 3 | Uptake of three gaseous CFC guests in cage 2 as a neat liquid. a, When neat liquid cage 2 was stirred under an atmosphere saturated with $\mathrm{CFCl}_{3}, \mathrm{CF}_{2} \mathrm{Cl}_{2}$, or $\mathrm{CF}_{3} \mathrm{Cl}$, uptake was observed of these gaseous guest molecules. The samples were subsequently analysed using solution-phase NMR techniques. b, Partial solution-phase ${ }^{1} \mathrm{H}$ NMR spectra $(298 \mathrm{~K}, 400 \mathrm{MHz}$, $\mathrm{CD}_{3} \mathrm{CN}$ ) of $\mathrm{CFC} \subset \mathbf{2}$ host-guest complexes, peaks correlated to bound guest are labelled with an asterisk (*), dotted lines have been added to help reference the free cage 2 peaks in the host-guest spectra. c, Partial solution-phase ${ }^{19} \mathrm{~F}$ NMR spectra (298 K, 377 $\mathrm{MHz}, \mathrm{CD}_{3} \mathrm{CN}$ ) of $\mathrm{CFC} \subset \mathbf{2}$ host-guest complexes, including a magnification of the bound guest peak. $\mathbf{d}$, The encapsulation of $\mathrm{CFCl}_{3}$ by cage 2 was reversible; $\mathrm{CFCl}_{3}$ could be removed from cage 2 under dynamic vacuum, and the cage could then be recycled. This process was monitored by ${ }^{19} \mathrm{~F}$ NMR.

\section{Conclusion}

Here we report the synthesis of a porous cage $\mathbf{2}$, a coordination cage that behaves as a liquid in the neat state. In addition to binding a wider range of guests than previously reported porous liquids, empty cage 2 could be recovered by releasing the encapsulated guests under reduced pressure, due to its own negligible vapour pressure. Consequently, this porous liquid is recyclable, an important consideration for the development of industrial materials. Future work will target the preparation of permanently porous liquids with still larger cavities, capable of binding more complex molecules as guests. While to the best of our 
knowledge coordination cages have not been previously used as scaffolds for porous liquids, supramolecular capsules have been constructed with a range of different sizes, geometries, and guest-binding preferences $^{21-40}$. By using coordination cages to build the cavities within porous liquids, we envisage new fluids with pores selected from the extensive library of existing coordination cages ${ }^{41-43}$. While the present system exhibits relatively weak guest binding, improvements to the binding strength of future generations of porous liquid coordination cages could potentially enable applications such as molecular separations ${ }^{44}$, extractions $^{45}$, and catalysis ${ }^{46-48}$, which have been already established for coordination cages. We envisage that such applications may ultimately be incorporated into flow systems of liquid cages based on existing cage frameworks.

Future generations of these materials may thus be used to repeatedly sequester and release sizecompatible guests from mixtures, rendering them useful in the context of chemical separations. Specific compounds might thus be isolated from mixtures through liquid-liquid extractions involving permanently porous ionic liquids. Their cavities offer a new route to selectivity beyond what is possible using current state-of-the-art ionic liquid extraction processes. The dynamic nature of coordination cages could thus yield new types of adaptable and stimuli-responsive materials ${ }^{49}$.

\section{Methods}

\section{Synthesis of cage 2 .}

Trialdehyde A (15.72 mg, 4 equiv.) and aniline $\mathbf{B}$ (156.00 mg, 12 equiv.) were combined in $5 \mathrm{~mL} \mathrm{CH}_{3} \mathrm{CN}$ and heated in a microwave reactor at $100{ }^{\circ} \mathrm{C}$ for 10 minutes. $\mathrm{Zn}\left(\mathrm{NTf}_{2}\right)_{2}(27.53 \mathrm{mg}, 4.4$ equiv.) was added then heated for an additional 10 minutes at $100^{\circ} \mathrm{C}$ in the microwave reactor. The cage was precipitated out of solution via the addition of diethyl ether $(5 \mathrm{~mL})$ and centrifuged. The resulting oil was concentrated under vacuum yielding a dark yellow, viscous liquid (172 mg, 88\% yield). ${ }^{1} \mathrm{H}$ NMR (400 MHz, $\left.\mathrm{CD}_{3} \mathrm{CN}\right): \delta=8.91$ $\left(\mathrm{s}, 1 \mathrm{H}, H_{a}\right), \delta=8.63\left(\mathrm{~s}, 2 \mathrm{H}, H_{b, c}\right), \delta=8.57\left(\mathrm{~d}, 1 \mathrm{H}, H_{d}\right), \delta=7.56\left(\mathrm{~s}, 1 \mathrm{H}, H_{e}\right), \delta=7.52\left(\mathrm{~s}, 1 \mathrm{H}, H_{f}\right), \delta=7.47(\mathrm{~s}$, $\left.1 \mathrm{H}, H_{g}\right), \delta=7.39\left(\mathrm{~s}, 1 \mathrm{H}, H_{h}\right), \delta=6.93\left(\mathrm{~d}, 2 \mathrm{H}, H_{i}\right), \delta=6.79\left(\mathrm{~d}, 2 \mathrm{H}, H_{j}\right), \delta=4.31\left(\mathrm{t}, 2 \mathrm{H}, H_{f}\right), \delta=4.16(\mathrm{t}, 2 \mathrm{H}$, $\left.H_{l}\right), \delta=3.87\left(\mathrm{~s}, 3 \mathrm{H}, H_{m}\right), \delta=3.58\left(\mathrm{~m}, 118 \mathrm{H}, \mathrm{H}_{\text {aliphatic } P E G}\right)$.

\section{Synthesis of guest $\subset 2$ in $\mathrm{CD}_{3} \mathrm{CN}$ solution.}

The guest $(10 \mu \mathrm{L})$ was added to a solution of cage $2\left(2.0 \mathrm{mM}\right.$ in $\left.\mathrm{CD}_{3} \mathrm{CN}, 0.5 \mathrm{~mL}\right)$ and the system equilibrated at $298 \mathrm{~K}$ for $18 \mathrm{~h} .{ }^{1} \mathrm{H}$ NMR $\left(400 \mathrm{MHz}, \mathrm{CD}_{3} \mathrm{CN}\right): t$-butanol $\subset \mathbf{2}, \delta=-1.43 \mathrm{ppm}$; $i$-butanol $\subset \mathbf{2}, \delta=$ $-1.35,-1.37$ ppm; $i$-propanol $\subset \mathbf{2}, \delta=-1.69,-1.71$ ppm; $s$-butanol $\subset \mathbf{2}, \delta=-1.43,-1.59$ ppm; $n$-propanol $\subset \mathbf{2}$, $\delta=-1.54,-1.71,-1.73$ ppm; $n$-butanol $\subset 2: \mathrm{N} / \mathrm{A} ; \mathrm{CFCl}_{3} \subset \mathbf{2}, \delta=8.86,8.63,8.59,8.55,8.53,7.56,7.45,7.44$, 7.44, 7.36, 7.35, 7.35, 6.91, 6.89, 6.72, 6.69, 4.29, 4.28, 4.27, 4.13, 3.85, 3.81, 3.79, 3.56, 3.55 ppm. ${ }^{19} \mathrm{~F}$ NMR (377 MHz, $\mathrm{CDCl}_{3}$, referenced to a $\mathrm{C}_{6} \mathrm{~F}_{6}$ capillary): $\mathrm{CFCl}_{3} \subset \mathbf{2}, \delta=-3.30 \mathrm{ppm}$. 


\section{Synthesis of alcohols $\subset 2$ under solvent free conditions.}

One alcohol isomer (10 wt $\%$ or $30 \mathrm{wt} \%)$ was added to a sample of neat liquid cage $2(\sim 100 \mathrm{mg})$. The mixture was stirred in a sealed system at $298 \mathrm{~K}$. The mixture was then transferred into a $3 \mathrm{mM}$ NMR tube, deposited in a centrifuge tube $(15 \mathrm{~mL})$ cushioned with cotton wool, and centrifuged at $3000 \mathrm{RPM}$ for 30 seconds. A $\mathrm{D}_{2} \mathrm{O}$ capillary was inserted into the NMR tube before being analysed by VT-NMR $(343 \mathrm{~K}) .{ }^{1} \mathrm{H}$ NMR (400 MHz, CD $\left.{ }_{3} \mathrm{CN}\right): t$-butanol $\subset \mathbf{2}, \delta=-0.57 \mathrm{ppm} ; i$-butanol $\subset \mathbf{2}, \delta=-0.79 \mathrm{ppm} ; i$-propanol $\subset \mathbf{2}, \delta=-$ 0.53, -0.68 ppm; $s$-butanol $\subset 2, \delta=-0.67,-0.77$ ppm; $n$-propanol $\subset 2, \delta=-0.56,-0.62,-0.82$ ppm; $n$-butanol $\subset 2$ : N/A.

\section{Synthesis of $\mathrm{CFC} \subset \mathbf{2}$ under solvent free conditions.}

Liquid $\mathrm{CFCl}_{3}(100 \mu \mathrm{L})$ was transferred into a large vial (4 dram) containing a smaller vial $(5 \mathrm{~mL})$ loaded with neat cage 2 (20 mg, $1 \mu \mathrm{mol}$ ) and a stir bar. The system was sealed with a Teflon screw cap and stirred at $298 \mathrm{~K}$ for $18 \mathrm{~h}$. After dissolving the residue contained in the inner vial in $\mathrm{CD}_{3} \mathrm{CN}(0.5 \mathrm{~mL})$ the sample was analysed via ${ }^{1} \mathrm{H}$ NMR and ${ }^{19} \mathrm{~F}$ NMR with a 1,3,5-tris(trifluoromethyl)benzene capillary in $\mathrm{CD}_{3} \mathrm{CN}$. ${ }^{1} \mathrm{H}$ NMR (400 MHz, $\left.\mathrm{CD}_{3} \mathrm{CN}\right): \delta=8.88,8.85,8.63,8.60,8.54,8.53,8.30$ (ref.), 7.56, 7.45, 7.44, 7.44, 7.36, $6.91,6.89,6.77,6.71,6.69,4.29,4.28,4.27,3.85,3.81,3.79,3.61,3.60,3.57,3.55,3.53 .{ }^{19} \mathrm{~F} \mathrm{NMR}(377$ $\left.\mathrm{MHz}, \mathrm{CD}_{3} \mathrm{CN}\right): \mathrm{CFCl}_{3} \subset \mathbf{2}, \delta=-3.30 \mathrm{ppm}$.

This experiment was repeated for $\mathrm{CF}_{2} \mathrm{Cl}_{2} \subset \mathbf{2}$ and $\mathrm{CF}_{3} \mathrm{Cl} \subset \mathbf{2}$ with the only modification being that the gaseous CFCs were introduced into a septum-sealed large vial by purging the atmosphere with the guest instead of transferring the CFC in the liquid state.

$\mathrm{CF}_{2} \mathrm{Cl}_{2} \subset 2:{ }^{1} \mathrm{H} \mathrm{NMR}\left(400 \mathrm{MHz}, \mathrm{CD}_{3} \mathrm{CN}\right) \delta=8.88,8.86,8.60,8.55,8.53,8.30$ (ref.), 7.54, 7.49, 7.45, 7.44, 7.44, 7.36, 6.91, 6.89, 6.77, 6.75, 6.73, 6.70, 4.29, 4.28, 4.27, 3.85, 3.81, 3.79, 3.78, 3.57, 3.55, 3.54. ${ }^{19} \mathrm{~F}$ NMR: $\mathrm{CF}_{2} \mathrm{Cl}_{2} \subset \mathbf{2}, \delta=-8.50 \mathrm{ppm}$.

$\mathrm{CF}_{3} \mathrm{Cl} \subset 2:{ }^{1} \mathrm{H} \mathrm{NMR}: \mathrm{N} / \mathrm{A} .{ }^{19} \mathrm{~F} \mathrm{NMR:} \mathrm{CF}_{3} \mathrm{Cl} \subset \mathbf{2}, \delta=-31.99 \mathrm{ppm}$.

\section{Recycling experiment of guest $\subset \mathbf{2}$.}

$T$-butanol $(10 \mu \mathrm{L})$ was added to a solution of cage $2\left(2.0 \mathrm{mM}\right.$ in $\left.\mathrm{CD}_{3} \mathrm{CN}, 0.5 \mathrm{~mL}\right)$ and the system equilibrated at $298 \mathrm{~K}$ for $18 \mathrm{~h}$. The sample was analysed by ${ }^{1} \mathrm{H}$ NMR before being placed under vacuum (1.35 mbar) for $1 \mathrm{~h}$. The residual material was dissolved in $\mathrm{CD}_{3} \mathrm{CN}$ and analysed by ${ }^{1} \mathrm{H} \mathrm{NMR}$. Upon confirmation that $t$-butanol was removed from the cavity of cage 2 , an additional $10 \mu \mathrm{L}$ of $t$-butanol was added to regenerate $t$-butanol $\subset \mathbf{2}$. This process was repeated 5 times. The recycling experiment was also performed with $\mathrm{CFCl}_{3}$ as the guest. For these experiments, the sample was also analysed via ${ }^{19} \mathrm{~F} \mathrm{NMR}$ in addition to ${ }^{1} \mathrm{H}$ NMR. 
Data availability statement

The authors declare that all data that supporting the findings of this study are included within the Article and its Supplementary Information, and are also available from the authors upon request. 


\section{References}

1. O’Reilly, N., Giri, N. \& James, S. L. Porous liquids. Chem. Eur. J. 13, 3020-3025 (2007).

2. Zhang, J. et al. Porous liquids: A promising class of media for gas separation. Angew. Chem. Int. Ed. 54, 932-936 (2015).

3. Li, P. et al. Electrostatic-Assisted Liquefaction of Porous Carbons. Angew. Chem. Int. Ed. 56, 1495814962 (2017).

4. Costa Gomes, M., Pison, L., Červinka, C. \& Padua, A. Porous ionic liquids or liquid metal-organic frameworks? Angew. Chem. Int. Ed. 57, 11909-11912 (2018).

5. Giri, N. et al. Liquids with permanent porosity. Nature 527, 216-220 (2015).

6. Shan, W. et al. New class of type III porous liquids: a promising platform for rational adjustment of gas sorption behavior. ACS Appl. Mater. Inter. 10, 32-36 (2018).

7. Liu, H. et al. A hybrid absorption-adsorption method to efficiently capture carbon. Nat. Commun. 5, 5147 (2014).

8. Gaillac, R. et al. Liquid metal-organic frameworks. Nat. Mater. 16, 1149-1155 (2017).

9. Bennett, T. D. \& Horike, S. Liquid, glass and amorphous solid states of coordination polymers and metal-organic frameworks. Nat. Rev. Mater. 3, 431-440 (2018).

10. Greenaway, R. L. et al. Understanding gas capacity, guest selectivity, and diffusion in porous liquids. Chem. Sci. 8, 2640-2651 (2017).

11. Zhang, F. et al. Thermodynamics and kinetics of gas storage in porous liquids. J. Phys. Chem. B 120, 7195-7200 (2016).

12. WBCSD \& WRI. A corporate accounting and reporting standard. Greenhouse Gas Protocol. https://ghgprotocol.org/sites/default/files/standards/ghg-protocol-revised.pdf (2012).

13. Castilla, A. M., Ronson, T. K. \& Nitschke, J. R. Sequence-dependent guest release triggered by orthogonal chemical signals. J. Am. Chem. Soc. 138, 2342-2351 (2016).

14. Tao, S. J. Positronium annihilation in molecular substances. J. Chem. Phys. 56, 5499-5510 (1972).

15. Kleywegt, G. J. \& Alwyn Jones, T. Detection, delineation, measurement and display of cavities in macromolecular structures. Acta Crystallogr. Sect. D Biol. Crystallogr. 50, 178-185 (1994).

16. Montzka, S. A. et al. An unexpected and persistent increase in global emissions of ozone-depleting CFC-11. Nature 557, 413-417 (2018).

17. Rigby, M. et al. Increase in CFC-11 emissions from eastern China based on atmospheric observations. Nature 569, 546-550 (2019).

18. Chen, T. H. et al. Mesoporous Fluorinated Metal-Organic Frameworks with Exceptional Adsorption of Fluorocarbons and CFCs. Angew. Chem. Int. Ed. 54, 13902-13906 (2015).

19. Shiflett, M. B. \& Yokozeki, A. Hydrogen substitution effect on the solubility of perhalogenated compounds in ionic liquid [bmim][PF6]. Fluid Phase Equilib. 259, 210-217 (2007).

20. Yang, D. et al. Encapsulation of Halocarbons in a Tetrahedral Anion Cage. Angew. Chem. Int. Ed. 54, 8658-8661 (2015). 
21. Heinz, T., Rudkevich, D. M. \& Rebek, J. Pairwise selection of guests in a cylindrical molecular capsule of nanometre dimensions. Nature 394, 764-766 (1998).

22. Akine, S., Miyashita, M. \& Nabeshima, T. A metallo-molecular cage that can close the apertures with coordination bonds. J. Am. Chem. Soc. 139, 4631-4634 (2017).

23. Käseborn, M., Holstein, J. J., Clever, G. H. \& Lützen, A. A rotaxane-like cage-in-ring structural motiffor a metallosupramolecular $\mathrm{Pd}_{6} \mathrm{~L}_{12}$ aggregate. Angew. Chem. Int. Ed. 57, 12171-12175 (2018).

24. Hof, F., Nuckolls, C. \& Rebek, J. Diversity and selection in self-assembled tetrameric capsules. J. Am. Chem. Soc. 122, 4251-4252 (2000).

25. Yan, Y. et al. Smart self-assemblies based on a surfactant-encapsulated photoresponsive polyoxometalate complex. Angew. Chem. Int. Ed. 49, 9233-9236 (2010).

26. Wang, M., Zheng, Y.-R., Ghosh, K. \& Stang, P. J. Metallosupramolecular tetragonal prisms via multicomponent coordination-driven template free self-assembly. J. Am. Chem. Soc. 132, 1-9 (2010).

27. Mondal, B. \& Mukherjee, P. S. Cage encapsulated gold nanoparticles as heterogeneous photocatalyst for facile and selective reduction of nitroarenes to azo compounds. J. Am. Chem. Soc. 140, 1259212601 (2018).

28. Bondy, C. R., Gale, P. A. \& Loeb, S. J. Metal-organic anion receptors: arranging urea hydrogen-bond donors to encapsulate sulfate ions. J. Am. Chem. Soc. 126, 5030-5031 (2004).

29. Scherer, M., Caulder, D. L., Johnson, D. W. \& Raymond, K. N. Triple helicate-tetrahedral cluster interconversion controlled by host-guest interactions. Angew. Chem. Int. Ed. 38, 1588-1592 (1999).

30. Liu, Y., Hu, C., Comotti, A. \& Ward, M. D. Supramolecular Archimedean cages assembled with 72 hydrogen bonds. Science 333, 436-440 (2011).

31. Ajami, D. \& Rebek, J. Compressed alkanes in reversible encapsulation complexes. Nat. Chem. 1, 8790 (2009).

32. Roy, X. et al. Prussian blue nanocontainers: Selectively permeable hollow metal-organic capsules from block ionomer emulsion-induced assembly. J. Am. Chem. Soc. 133, 8420-8423 (2011).

33. Campos-Fernández, C. S. et al. Anion template effect on the self-assembly and interconversion of metallacyclophanes. J. Am. Chem. Soc. 127, 12909-12923 (2005).

34. Turunen, L., Warzok, U., Schalley, C. A. \& Rissanen, K. Nano-sized $\mathrm{I}_{12} \mathrm{~L}_{6}$ molecular capsules based on the $\left[\mathrm{N} \cdots \mathrm{I}^{+} \cdots \mathrm{N}\right]$ halogen bond. Chem 3, 861-869 (2017).

35. Sun, L. Y. et al. Template synthesis of three-dimensional hexakisimidazolium cages. Angew. Chem. Int. Ed. 57, 5161-5165 (2018).

36. Markiewicz, G. et al. Selective $\mathrm{C}_{70}$ encapsulation by a robust octameric nanospheroid held together by 48 cooperative hydrogen bonds. Nat. Commun. 8, 15109 (2017).

37. Jie, K. et al. Linear positional isomer sorting in nonporous adaptive crystals of a pillar[5]arene. J. Am. Chem. Soc. 140, 3190-3193 (2018).

38. Jia, C. et al. Selective binding of choline by a phosphate-coordination-based triple helicate featuring an aromatic box. Nat. Commun. 8, 938 (2017). 
39. Brachvogel, R. C., Hampel, F. \& Von Delius, M. Self-assembly of dynamic orthoester cryptates. Nat. Commun. 6, 7129 (2015).

40. Ozores, H. L., Amorín, M. \& Granja, J. R. Self-assembling molecular capsules based on $\alpha, \gamma$-cyclic peptides. J. Am. Chem. Soc. 139, 776-784 (2017).

41. Xuan, W. et al. A chiral quadruple-stranded helicate cage for enantioselective recognition and separation. J. Am. Chem. Soc. 134, 6904-6907 (2012).

42. Preston, D., Sutton, J. J., Gordon, K. C. \& Crowley, J. D. A nona-nuclear heterometallic $\mathrm{Pd}_{3} \mathrm{Pt}_{6}$ “donut"-shaped cage: molecular recognition and photocatalysis. Angew. Chem. Int. Ed. 57, 86598663 (2018).

43. Chepelin, O. et al. Luminescent, enantiopure, phenylatopyridine iridium-based coordination capsules. J. Am. Chem. Soc. 134, 19334-19337 (2012).

44. Krieg, E. et al. A recyclable supramolecular membrane for size-selective separation of nanoparticles. Nat. Nanotechnol. 6, 141-146 (2011).

45. Lee, D. W. et al. Supramolecular fishing for plasma membrane proteins using an ultrastable synthetic host-guest binding pair. Nat. Chem. 3, 154-159 (2011).

46. Yoshizawa, M., Tamura, M. \& Fujita, M. Diels-alder in aqueous molecular hosts: unusual regioselectivity and efficient catalysis. Science 312, 251-254 (2006).

47. Cullen, W. et al. Highly efficient catalysis of the Kemp elimination in the cavity of a cubic coordination cage. Nat. Chem. 8, 231-236 (2016).

48. Zhang, Q., Catti, L., Pleiss, J. \& Tiefenbacher, K. Terpene cyclizations inside a supramolecular catalyst: leaving-group-controlled product selectivity and mechanistic studies. J. Am. Chem. Soc. 139, 11482-11492 (2017).

49. Fukui, T. Control over differentiation of a metastable supramolecular assembly in one and two dimensions. Nat. Chem. 9, 493-499 (2017).

\section{Acknowledgments}

L.M., A.B.G., C.J.E.H., and A.T. acknowledge support from the UK Engineering and Physical Sciences Research Council (EPSRC EP/P027067/1) and the European Research Council (ERC 695009). C.C.P. (EPSRC DTP grant EP/M508007/1) acknowledges the Engineering and Physical Sciences Research Council for funding. L.L. acknowledges an EPSRC Departmental Studentship. A.W. and A.R.S. acknowledge the National Centre for Research and Development for funding (LIDER/024/391/L-5/13/NCBR/2014 and PRELUDIUM UMO-2016/21/N/ST5/00851). T.D.B. would like to thank the Royal Society for a University Research Fellowship (UF150021), and for a Research Grant (RSGIR1\180395). The authors acknowledge Zhichen Wu and Ophélie Planes for preliminary work done on this project as well as David S. Robson for videography. Additionally, the authors would like to thank the NMR facility at the University of Cambridge Chemistry Department and the EPSRC UK National Mass Spectrometry Facility at Swansea University for characterization. 


\section{Author contributions}

L.M., A.B.G, C.J.E.H. and J.RN. conceived and designed the experiments. A.T. designed the ligand synthesis. L.M., C.J.E.H., and A.W. performed the synthetic work. C.C.P. conducted and analysed all rheological measurements. T.D.B. and L.L. performed and analysed DSC and TGA measurements. L.M. led the project overall. All authors contributed to the manuscript preparation.

\section{Competing Interests}

The authors declare no competing interests. 